\title{
Detection of Jaagsiekte sheep retrovirus in apparently healthy sheep by real-time TaqMan PCR in comparison with histopathological findings
}

\author{
Aliasghar Bahari ${ }^{1}$, Masoud Sabouri Ghannad ${ }^{2}$, Omid Dezfoulian $^{3}$, \\ Fereydon Rezazadeh ${ }^{4}$, Ali Sadeghi-Nasab ${ }^{1}$ \\ ${ }^{1}$ Department of Clinical Sciences, Faculty of Veterinary Science, \\ Bu-Ali Sina University, 65176-58978 Hamedan, Iran \\ ${ }^{2}$ Department of Microbiology, Faculty of Medicine, \\ Hamedan University of Medical Sciences, 65176-19654 Hamedan, Iran \\ ${ }^{3}$ Department of Pathobiology, Faculty of Veterinary Medicine, \\ Lorestan University, 68137-17133 Khoramabad, Iran \\ ${ }^{4}$ Department of Clinical Sciences, Faculty of Veterinary Medicine, \\ Tabriz University, 51666-16471 Tabriz, Iran \\ aliasghar.bahari@basu.ac.ir \\ Received: November 3, 2015 Accepted: February 15, 2016
}

\begin{abstract}
Introduction: The aim of this study was to use TaqMan real-time PCR technique to investigate Jaagsiekte sheep retrovirus (JSRV) proviral DNA in whole blood samples of sheep, and compare the results to those of histopathological examinations. Material and Methods: Eighty blood samples from clinically healthy sheep were randomly collected before the animals were slaughtered. Ten tissue samples from each lung and associated caudal mediastinal lymph node were taken. Results: Fifteen (18.75\%) blood samples were found to contain proviral DNA, and $11(13.75 \%)$ corresponding lung samples showed microscopic changes consistent with ovine pulmonary adenocarcinoma. None of the samples displayed metastases to the caudal mediastinal lymph nodes. The prominent pattern of neoplastic nodules consisted of acinar (alveolar) form. Conclusion: The results indicated the higher sensitivity of real-time PCR compared to histopathological examinations in detection of ovine pulmonary adenocarcinoma.
\end{abstract}

Keywords: sheep, ovine pulmonary adenocarcinoma, Jaagsiekte retrovirus, real-time PCR, histopathology.

\section{Introduction}

Ovine pulmonary adenocarcinoma (OPA), which is also known as sheep pulmonary adenomatosis (SPA) or Jaagsiekte, is an important infectious disease of sheep that has been reported in many sheep-rearing countries of Europe, Africa, America, and Asia (28). OPA is a contagious tumour arising from alveolar type II pneumocytes and rarely from non-ciliated bronchiolar (Clara) cells. Tumour metastases can be found in thoracic and occasionally in extrathoracic tissues $(6,7,8$, $22,25)$. The disease is caused by an exogenous $\beta$-retrovirus, known as Jaagsiekte sheep retrovirus (JSRV), which induces oncogenic transformation of alveolar and bronchiolar secretory epithelial cells (21). Clinical signs of the disease can include respiratory distress and emaciation. The viral infection may increase the animal susceptibility to secondary bacterial infections in the lungs. Death may occur within a few weeks after the onset of symptoms $(5,19)$. The most probable mode of transmission of JSRV is inhalation of aerosols from affected flock mates (28). OPA occurs naturally in sheep and rarely in goats (21). Although the disease may affect lambs as young as two months old, sheep most often show clinical features at 2-4 years of age $(8,12,28)$. In some affected flocks, the annual mortality rate due to the disease may be as high as $10 \%-$ $20 \%$, although the rate of $1 \%-3 \%$ is more common (28). 
Currently, there is no treatment or vaccination available for JSRV infection and clinical OPA which is inevitably fatal. OPA can cause substantial losses in affected flocks, therefore, in order to prevent the spread of JSRV infection, a reliable diagnostic test for detection of infected sheep is needed (16). Diagnosis of Jaagsiekte is based on clinical signs and post mortem examination and there are no available cost-effective serological assays for detection of JSRV infection, since the virus does not induce a specific antibody response in infected animals $(20,29)$. However, there are other diagnostic methods, such as thoracic radiography (30), ultrasonography (27), lung biopsy $(1,23)$ followed by histopathological examination (2). These methods lack sufficient sensitivity or specificity to detect viral infections, especially in early stages of the disease. Nevertheless, a specific PCR technique has been developed for detection of JSRV proviral DNA in tissues of the lymphoreticular system and peripheral blood mononuclear cells (21). Improved PCR techniques have demonstrated JSRV proviral DNA prior to the onset of neoplasia in experimentally inoculated lambs (12) and during the pre-clinical period of the natural disease in commercial sheep flocks (9). This study was conducted to investigate the presence of JSRV proviral DNA in the blood samples of apparently healthy sheep using TaqMan real-time PCR as a highly sensitive method. The results of the real-time PCR assay were compared with those of histopathological examination of lung tissue.

\section{Material and Methods}

Preparation of blood samples. Eighty clinically healthy sheep were randomly chosen for sampling at Hamedan industrial abattoir, located in central-west Iran. Blood samples were taken into $10 \mathrm{~mL}$ EDTA vacutainer blood collection tubes through jugular venipuncture before the animals were slaughtered. Whole blood samples were stored at $-70^{\circ} \mathrm{C}$ until DNA extraction.

Preparation of lung samples. Immediately after slaughtering, the lungs were removed and examined carefully for visible lesions of OPA. Lung tissue samples of $1 \mathrm{~cm}^{3}$ of size from 10 fixed locations (Fig. 1) and the caudal mediastinal lymph nodes (MLNs) were collected. The tissues were fixed in $10 \%$ neutral buffered formalin and embedded in paraffin. Haematoxylin and eosin (H\&E) stained 4-6 $\mu \mathrm{m}$ thick sections were examined histopathologically.

DNA extraction and real-time PCR. DNA extraction from blood samples was performed using QIAamp DNA Blood Mini Kit (Qiagen, Germany) according to the manufacturer's instruction, and the collected DNA samples were subjected to real-time PCR to detect JSRV proviral DNA. A previously designed TaqMan probe based on JSRV LTR sequence and a pair of primers to target a specific conserved 176 base pair region within the JSRV LTR sequence were used (15).
The Quanti Tect Probe PCR kit (Qiagen, Germany) was applied with $0.9 \mu \mathrm{M}$ of primers P1 (5'-TGG GAG CTC TTT GGC AGA AGC C-3') and P3 (5'-CAC CGG ATT CTT ACA CAA TCA CCG G-3') (21), $0.2 \mu \mathrm{M}$ probe (5'-FAM-AGC TCC CTG TCC CGC CAC CCT C-3' BHQ1) (15), and $0.5 \mu \mathrm{L}$ of template DNA in a total reaction volume of $25 \mu \mathrm{L}$.

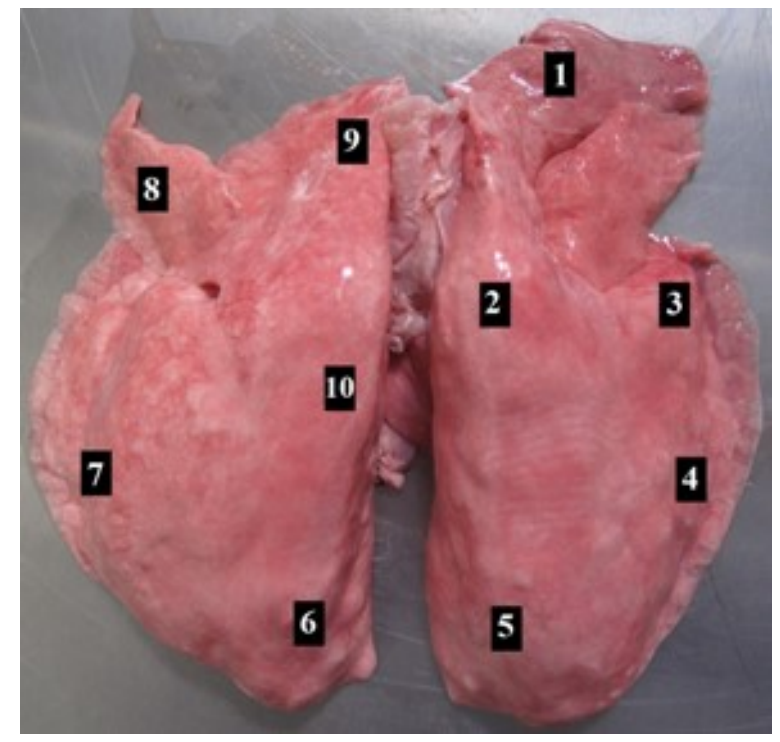

Fig. 1. Sampling points in the lungs

Amplification was carried out by a CFX 96 thermocycler (Bio Rad, USA) using the following settings: initial denaturation at $95^{\circ} \mathrm{C}$ for $15 \mathrm{~min}$ (one cycle), denaturation at $94^{\circ} \mathrm{C}$ for $15 \mathrm{~s}$, and extension at $60^{\circ} \mathrm{C}$ for $1 \mathrm{~min}(45$ cycles). The analysis of the amplification reaction products was performed using the software associated with the instrument (CFX manager Software). Plasmid pJS21-VR3-U3, carrying nucleotides 5350-7383 of JSRV genome (Genbank AF105220) inserted into pGEM-T plasmid, was used as positive control in the assay.

\section{Results}

Fifteen out of $80(18.75 \%)$ blood samples were positive for JSRV proviral DNA by TaqMan real-time PCR method and $11(13.75 \%)$ of corresponding lung samples showed microscopic changes consistent with OPA. Microscopic examination showed no metastases to the caudal MLNs. Tumour distribution patterns in pulmonary tissues were variable. The prominent pattern of neoplastic nodules consisted of acinar (alveolar) and to a lesser extent the other forms were present, such as papillary, adenomatous, bronchiolar, and mixed features (Figs 2-6). Tumour cells in acinar pattern were chiefly composed of single and rarely multiple layers of cuboidal to short columnar epithelial cells, whereas the bronchioles were lined predominantly by tall columnar cells. The nodular lesions were frequently surrounded by expanded rims of fibrous connective tissue, which were 


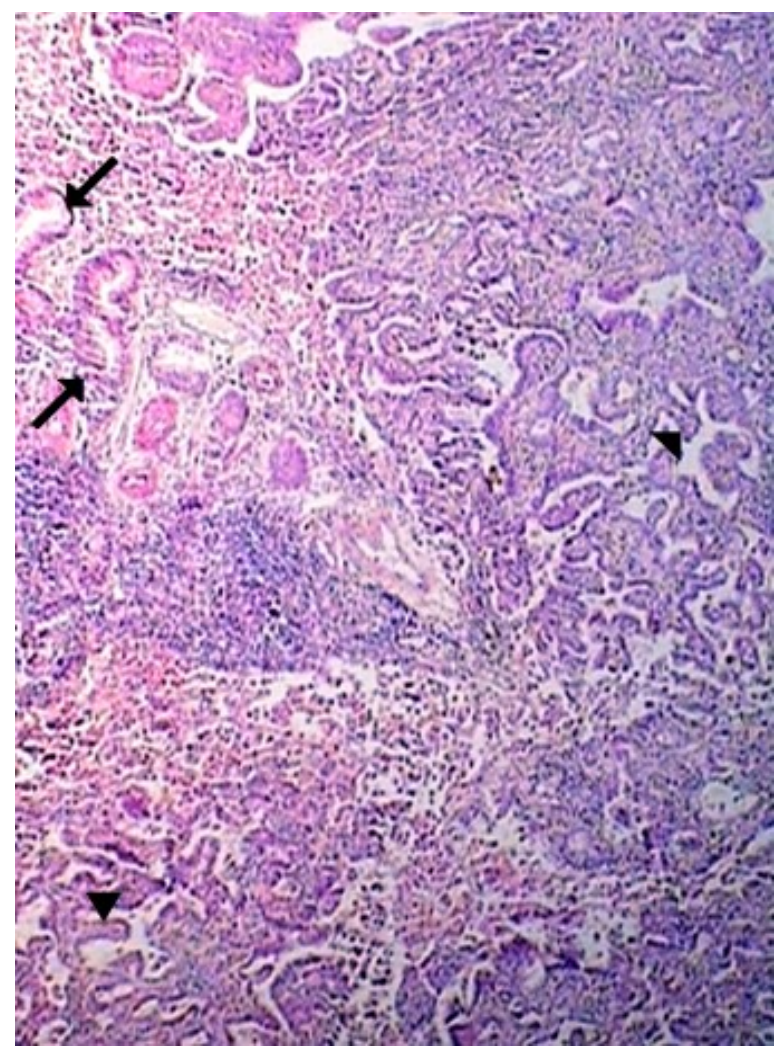

Fig. 2. Mixed adenocarcinoma of alveolar (arrows) and papillary (arrow heads) patterns. Aggregation of mononuclear cells is evident (H\&E, 100×)

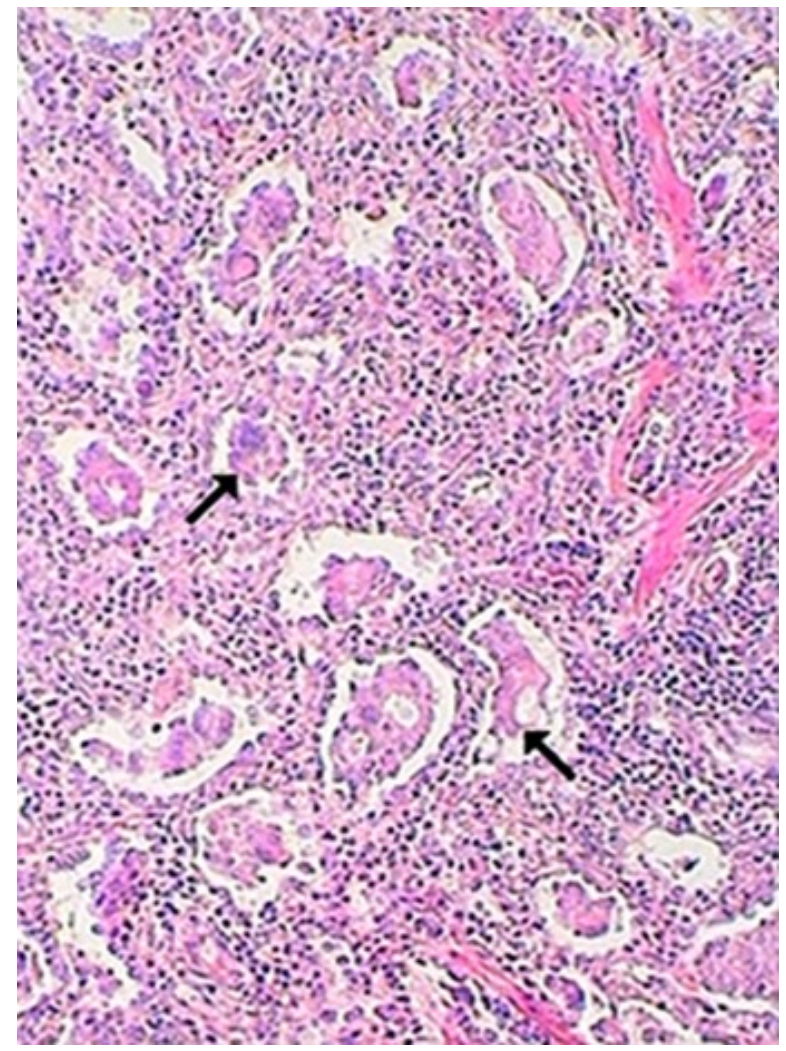

Fig. 3. Adenomatous pattern (arrows) of pulmonary adenocarcinoma with high population of mononuclear cells (H\&E, 400×)

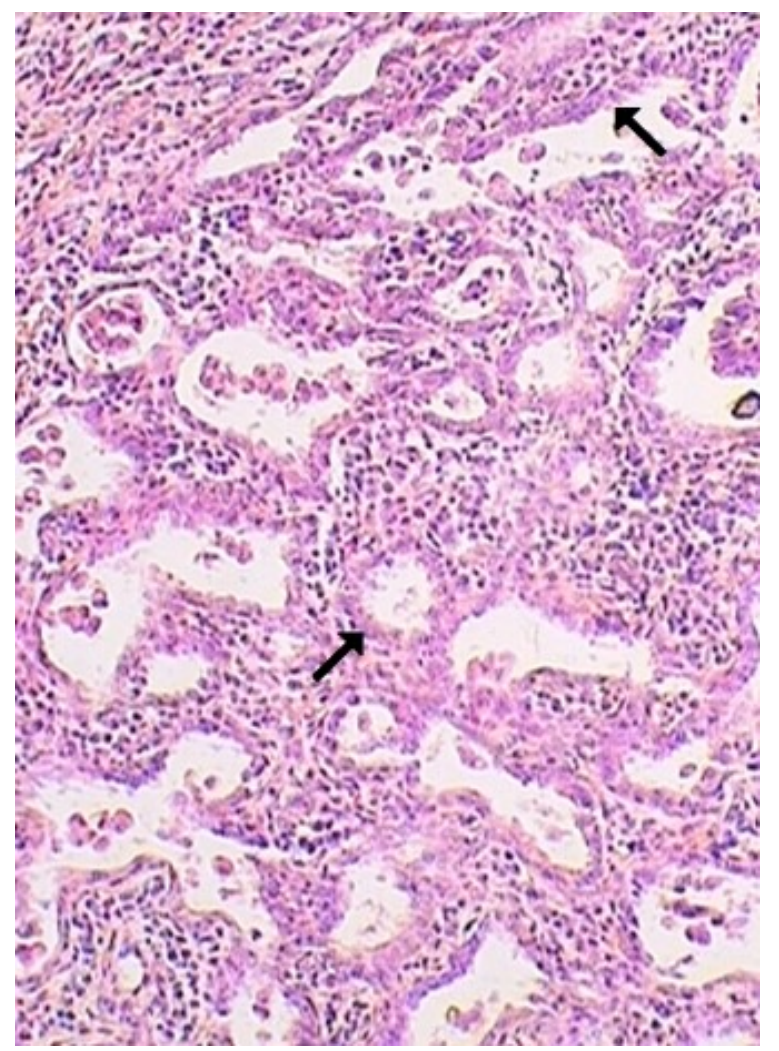

Fig. 4. Alveolar or acinar formation of pulmonary adenocarcinoma (arrows). The neoplastic alveoli are separated by thick fibrous connective tissue walls $(\mathrm{H} \& \mathrm{E}, 400 \times)$

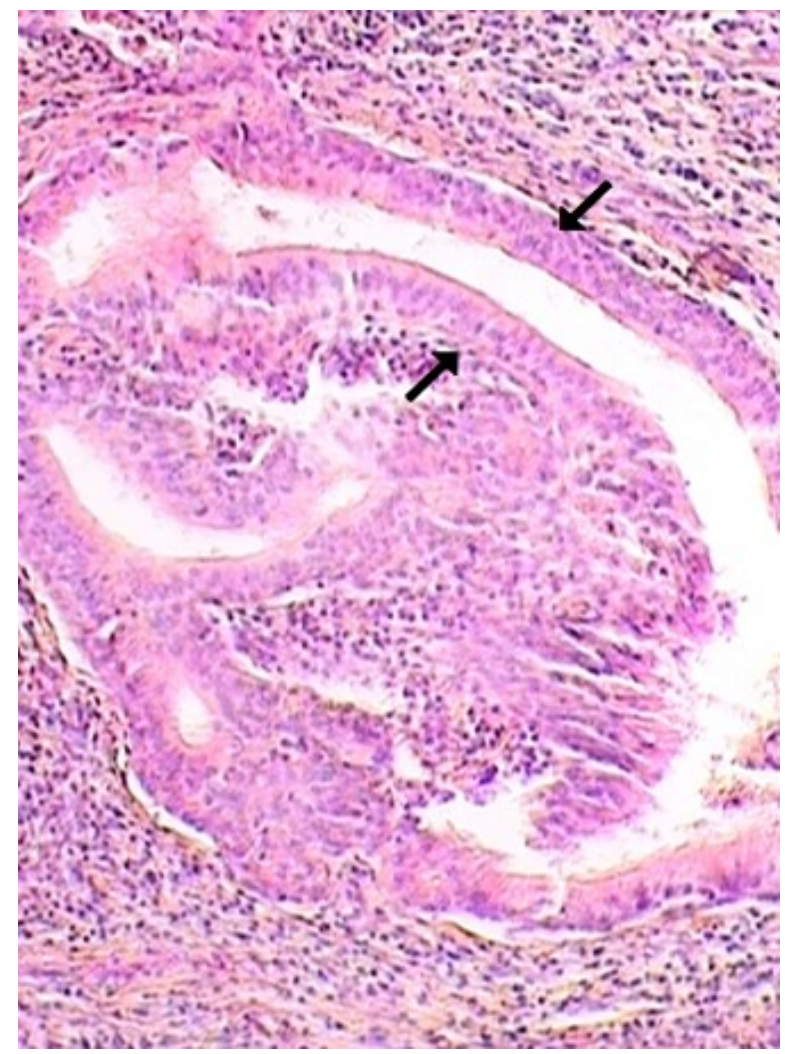

Fig. 5. Bronchiolar morphology of pulmonary adenocarcinoma. The bronchioles are lined with high columnar epithelial cells (arrows) (H\&E, 400×) 
invaded by high population of mononuclear cells. In some cases, large foci of proliferated lymphoid cells were seen around the bronchioles or affected interstitial tissue. The detailed results of real-time PCR and histopathological examinations are summarised in Table 1.

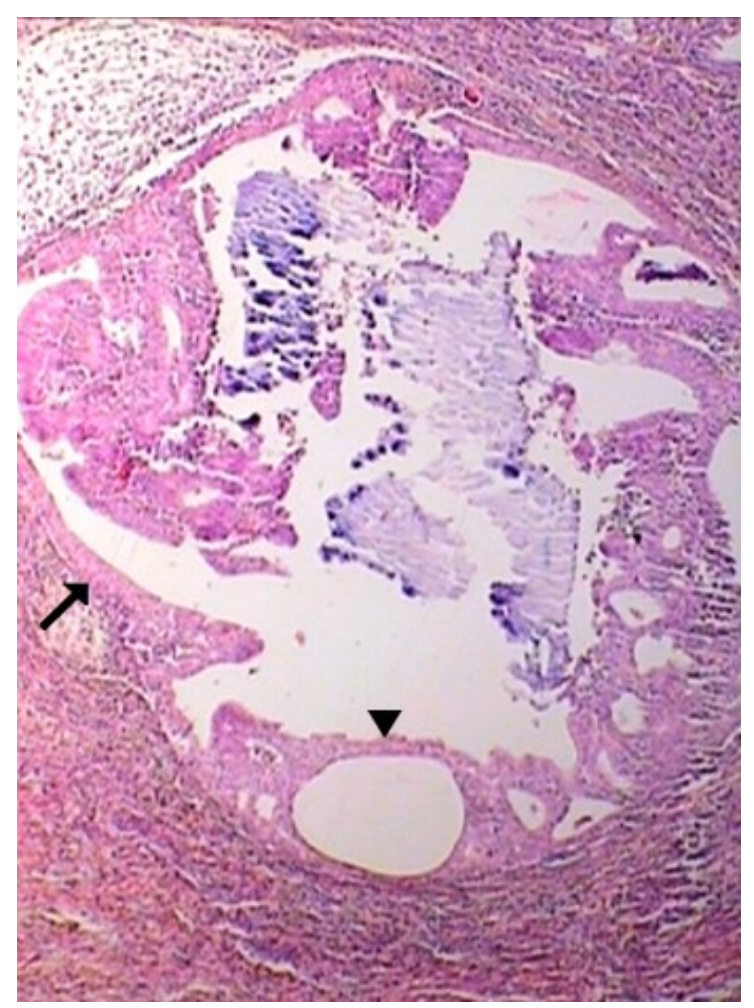

Fig. 6. Bronchiolar (arrow) and alveolar (arrow head) growth patterns of the tumour $(\mathrm{H} \& \mathrm{E}, 100 \times)$

\section{Discussion}

OPA is an important contagious viral disease of sheep in many sheep-rearing countries (28). Eliminating the disease from a flock is difficult, because there is no available cost-effective assay for the detection of JSRV infection and most of the diagnostic tests cannot detect infection in the preclinical stage.

Only few studies have been performed to detect
JSRV infection and estimate the prevalence of the disease worldwide, and the economic impact of the disease is also not clear. For example, Mackay and Nisbet (18) reported grossly visible OPA lesions in 52 out of $280,000(0.02 \%)$ slaughtered sheep, whereas Cousens et al. (4) found 31 positive cases among 3385 $(0.9 \%)$ cull sheep examined using histopathological methods. Khodakaram-Tafti and Razavi (14) examined morphopathologically lung samples of slaughtered sheep from Fars province of Iran and identified OPA in 21 $(0.22 \%)$ cases. Rezazadeh et al. (24) reported the prevalence rate of $2.57 \%$ for OPA based on a study of 468 lung samples of slaughtered animals in the suburbs of Tabriz. In another study, Khodakaram-Tafti et al. (13) performed macroscopic and histopathological examinations as well as the JSRV detection by RT-PCR in lung samples from 10 spontaneously OPA-affected Iranian crossbred sheep from herds of Fars province and from 10 apparently unaffected animals in a close contact with affected animals in the same herds. Neoplastic lesions were observed in lung samples of all affected sheep and the presence of pulmonary adenocarcinoma was histopathologically confirmed. Furthermore, 100\% of the lungs were found JSRV positive by RT-PCR method. No relevant lesions were detected in the lungs of the 10 apparently healthy sheep having had contact with affected animals; however, positive PCR results were observed in four cases. Gonzalez et al. (9) found JSRV proviral DNA in grossly normal lungs and peripheral blood leuckocytes of apparently healthy sheep. However, low sensitivity of traditional PCR in JSRV detection of blood samples at the level of individual animals has been noted (16). When comparing the sensitivity of real-time PCR and heminested PCR (hnPCR) methods, Kycko and Reichert (15) found that the implementation of real-time PCR method is a good alternative to the traditional PCR and hnPCR in JSRV detection and, apart from histopathological and immunohistochemical examinations, may be used to confirm the infection in clinically suspected animals, or employed as a screening method in control or eradication programmes.

Table 1. Characteristics of lung samples positive in real-time PCR and histopathological examination

\begin{tabular}{llllll}
\hline \multirow{2}{*}{ Case No. } & Sex & Age (year) & \multicolumn{2}{l}{ Real-time PCR } & \multicolumn{2}{l}{ Histopathology } \\
\cline { 3 - 6 } & & & Ct value & Location & Pattern \\
\hline 16 & M & 3 & 25 & L5 & Adenocarcinoma \\
\hline 20 & M & 2 & 28 & L5 & Alveolar \\
\hline 34 & M & 1 & 34 & L3, L8 & Early stage \\
\hline 35 & M & 1 & 37 & L4 & Early stage \\
\hline 37 & M & 2 & 39 & L3 & Early stage \\
\hline 39 & F & 4 & 24 & L3, L6, L8 & Alveolar \\
\hline 44 & M & 2 & 28 & L6 & Adenocarcinoma \\
\hline 46 & M & 1 & 27 & L3 & Alveolar \\
\hline 50 & M & 1 & 31 & L9 & Alveolar \\
\hline 68 & M & 2 & 40 & L4 & Early stage \\
\hline 69 & M & 2 & 27 & L3 & Adenocarcinoma \\
\hline
\end{tabular}


The advantages of TaqMan real-time PCR method over the conventional PCR assay are generally approved as real-time PCR is a more sensitive and specific method with a much shorter detection time. Detection and analysis of real-time PCR product is performed simultaneously during the amplification process by the software, whereas in the case of traditional PCR, the analysis of the results requires an additional step of agarose gel electrophoresis using factors such as ethidium bromide and UV light, which can be hazardous for human health $(11,17)$. In the current research, TaqMan real-time PCR technique allowed detecting JSRV proviral DNA in $18.75 \%$ (15 out of 80) of blood samples which showed a high infection rate in apparently healthy sheep. In addition, the results of this study showed that the rate of viral infection detected by real-time PCR is much higher compared to histopathological examination. This is in agreement with previous reports demonstrating that the number of JSRV infected animals is much higher than the number that will ever develop the disease $(3,10$, 26). In addition, histopathological lesions were found in 11 out of 15 PCR positive samples, indicating higher sensitivity of real-time PCR.

The presence of viral infection in Hamedan region suggests that more studies should be conducted to determine the exact prevalence of the disease and define economic losses due to JSRV infection. It seems that PCR and/or real-time PCR should be considered as suitable diagnostic methods for this purpose. Such valuable data can then be used to select appropriate strategies for preventing, controlling, and eradicating the disease.

Conflict of Interests Statement: The authors declare that there is no conflict of interests regarding the publication of this article.

Financial Disclosure Statement: This study was supported by the Iran National Science Foundation (No. 87041893).

Animal Rights Statement: The experiment was approved by the Ethics Committee of Bu-Ali Sina University, Hamedan-Iran.

Acknowledgements: The authors thank Christina Cousens, Moredun Research Institute, UK, for kindly providing the pGEM-T plasmid.

\section{References}

1. Begin R., Rola-Pleszczynski M., Sirois P., Masse S., Nadeau D., Bureau M.A.: Sequential analysis of the bronchoalveolar milieu in conscious sheep. J Appl Physiol 1981, 50, 665-671.

2. Beytut E., Sözmen M., Erginsoy S.: Immunohistochemical detection of pulmonary surfactant proteins and retroviral antigens in the lungs of sheep with pulmonary adenomatosis. J Comp Pathol 2009, 140, 43-53.
3. Caporale M., Centorame P., Giovannini A., Sacchini F., Di Ventura M., De las Heras M., Palmarini M.: Infection of lung epithelial cells and induction of pulmonary adenocarcinoma is not the most common outcome of naturally occurring JSRV infection during the commercial lifespan of sheep. Virology 2005, 338, 144-153.

4. Cousens C., Gibson L., Finlayson J., Pritchard I., Dagleish M.P.: Prevalence of ovine pulmonary adenocarcinoma (Jaagsiekte) in a UK slaughterhouse sheep study. Vet Rec 2015, 176, 413.

5. De las Heras M., Gonzalez L., Sharp J.M.: Pathology of ovine pulmonary adenocarcinoma. Curr Top Microbiol Immunol 2003, 275, 25-54.

6. De Martini J.C., Rosadio R.H., Lairmore M.D.: The etiology and pathogenesis of ovine pulmonary carcinoma (sheep pulmonary adenomatosis). Vet Microbiol 1988, 17, 219-236.

7. De Martini J.C., York D.F.: Retrovirus-associated neoplasms of the respiratory system of sheep and goats. Ovine pulmonary carcinoma and enzootic nasal tumor. Vet Clin North Am Food Anim Pract 1997, 13, 55-70.

8. Garcia-Goti M., Gonzalez L., Cousens C., Cortabarria N., Extramiana A.B., Minguijon E., Ortin A., De las Heras M., Sharp J.M.: Sheep pulmonary adenomatosis: characterization of two pathological forms associated with jaagsiekte retrovirus. J Comp Pathol 2000, 122, 55-65.

9. Gonzalez L., Garcia-Goti M., Cousens C., Dewar P., Cortabarria N., Extramiana A.B., Ortin A., De Las Heras M., Sharp J.M.: Jaagsiekte sheep retrovirus can be detected in the peripheral blood during the pre-clinical period of sheep pulmonary adenomatosis. J Gen Virol 2001, 82, 1355-1358.

10. Griffiths D.J., Martineau H.M., Cousens C.: Pathology and pathogenesis of ovine pulmonary adenocarcinoma. J Comp Pathol 2010, 142, 260-283.

11. Hoffmann B., Beer M., Reid S.M., Mertens P., Oura C.A., Van Rijn P.A., Slomka M.J., Banks J., Brown I.H., Alexander D.J., King D.P.: A review of RT-PCR technologies used in veterinary virology and disease control: sensitive and specific diagnosis of five livestock diseases notifiable to the World Organisation for Animal Health. Vet Microbiol 2009, 139, 1-23.

12. Holland M.J., Palmarini M., Garcia-Goti M., Gonzalez L., McKendrick I., De Las Heras M., Sharp J.M.: Jaagsiekte retrovirus is widely distributed both in $\mathrm{T}$ and $\mathrm{B}$ lymphocytes and in mononuclear phagocytes of sheep with naturally and experimentally acquired pulmonary adenomatosis. J Virol 1999, 73, 4004-4008.

13. Khodakaram-Tafti A., Mohammadi A., Mansourian M.: Detection of the Jaagsiekte Sheep Retrovirus by RT-PCR in naturally ovine pulmonary adenocarcinoma-affected sheep and in apparently healthy in contact-sheep. Rev Med Vet 2009, 160, 319-323.

14. Khodakaram-Tafti A., Razavi Z.: Morphopathological study of naturally occurring ovine pulmonary adenocarcinoma in sheep in Fars province, Iran. Iranian J Vet Res 2010, 11, 134-138.

15. Kycko A., Reichert M.: PCR-based methods for detection of JSRV in experimentally and naturally infected sheep. Bull Vet Inst Pulawy 2010, 54, 445-450.

16. Lewis F.I., Brülisauer F., Cousens C., McKendrick I.J., Gunn G.J.: Diagnostic accuracy of PCR for jaagsiekte sheep retrovirus using field data from 125 Scottish sheep flocks. Vet J 2011, 187, 104-108.

17. Mackay J.M., Arden K.E., Nitsche A.: Real-time PCR in virology. Nucleic Acids Res 2002, 30, 1292-1305.

18. Mackay J.M., Nisbet D.I.: Jaagsiekte - a hazard of intensified sheep husbandry. Vet Rec 1966, 78, 18-24.

19. Nisbet D.I., Mackay J.M., Smith W., Gray E.W.: Ultrastructure of sheep pulmonary adenomatosis (Jaagsiekte). J Pathol 1971,103, 157-162.

20. Ortin A., Minguijon E., Dewar P., Garcia M., Ferrer L.M., Palmarini M., Gonzalez L., Sharp J.M., De Las Heras M.: Lack of a specific immune response against a recombinant capsid protein of jaagsiekte sheep retrovirus in sheep and goats 
naturally affected by enzootic nasal tumour or sheep pulmonary adenomatosis. Vet Immunol Immunopathol 1998, 61, 229-237.

21. Palmarini M., Sharp J.M., De Las Heras M., Fan H.: Jaagsiekte sheep retrovirus is necessary and sufficient to induce a contagious lung cancer in sheep. J Virol 1999, 73, 6964-6972.

22. Platt J.A., Kraipowich N., Villafane F.A., De Martini J.C.: Alveolar type II cells expressing jaagsiekte sheep retrovirus capsid protein and surfactant proteins are the predominant neoplastic cell type in ovine pulmonary adenocarcinoma. Vet Pathol 2002, 39, 341-352.

23. Pusterla N., Braun U., Grest P., Ossent P.: Ein Fall von Lungenadenomatose bei einem Schaf: Diagnose durch Lungenbiopsie unter Ultraschallkontrolle. Tierärztl Umschau 1995, 50, 340-343.

24. Rezazadeh F., Zarrini G.H., Cousens C., Attarilar N.: Prevalence of jaagsiekte sheep retrovirus infection in North-West Iran. Global Vet 2012, 9, 535-540.

25. Rosadio R.H., Sharp J.M., Lairmore M.D., Dahlberg J.E., De Martini J.C.: Lesions and retroviruses associated with naturally occurring ovine pulmonary carcinoma (sheep pulmonary adenomatosis). Vet Pathol 1988, 25, 58-66.

26. Salvatori D., Gonzalez L., Dewar P., Cousens C., De Las Heras M., Dalziel R.G., Sharp J.M.: Successful induction of ovine pulmonary adenocarcinoma in lambs of different ages and detection of viraemia during the preclinical period. J Gen Virol 2004, 85, 3319-3324.

27. Scott P.R., Gessert M.E.: Ultrasonographic examination of the ovine thorax. Vet J 1998, 155, 305-310.

28. Sharp J.M., De Martini J.: Natural history of JSRV in sheep. Curr Top Microbiol Immunol 2003, 275, 55-79.

29. Sharp J.M., Herring A.: Sheep pulmonary adenomatosis: demonstration of a protein which crossreacts with the major core proteins of Mason-Pfizer monkey virus and mouse mammary tumour virus. J Gen Virol 1983, 64, 2323-2327.

30. Wyn-Jones G., Clarkson M.J.: Radiologic detection of ovine hydatidosis. Vet Radiol 1984, 25, 182-186. 